\title{
AVALIAÇÃO NUMÉRICA DA FLUIDODINÂMICA EM BIORREATOR EM ESCALA DE BANCADA PARA O CULTIVO DE MICROALGAS
}

\author{
L. GERMER ${ }^{1}$, L. T. PEREIRA ${ }^{1}$, C. K. SOUZA ${ }^{1}$, L. F. CARVALHO, L. M. ROSA ${ }^{1}$ \\ ${ }^{1}$ Universidade Regional de Blumenau, Departamento de Engenharia Química \\ E-mail para contato: leorosa@gmail.com
}

\begin{abstract}
RESUMO - Microalgas são organismos capazes de auxiliar na produção de diversos compostos. No entanto, a viabilidade econômica de processos empregando microalgas é questionada. Seu cultivo demanda atenção e cautela para características como a exposição a tensões cisalhantes elevadas, iluminação inadequada e baixo contato com o substrato. No presente estudo, são apresentadas avaliações numéricas da fluidodinâmica presente em fotobiorreator em escala de bancada, com capacidade de 2 L. Foi utilizada a técnica da fluidodinâmica computacional para estimar o escoamento monofásico, turbulento, em uma geometria axissimétrica. Observou-se que o sistema possui condições propícias à cultura de microalgas, exceto em regiões próximas à alimentação, nas quais se observaram valores elevados para a tensão de cisalhamento.
\end{abstract}

\section{INTRODUÇÃO}

Há um considerável interesse no uso de microalgas para as mais diversas finalidades, tais como produção de fármacos, cosméticos, alimentos, produtos químicos e biocombustíveis (Nikolaou et al., 2016). Seu cultivo, no entanto, demanda atenção e cautela para com algumas peculiaridades do processo, tais como a exposição a tensões cisalhantes elevadas, iluminação inadequada e baixo contato com o substrato.

Milledge (2011) destaca que, embora haja motivação para a produção de biocombustíveis a partir de microalgas, sua viabilidade econômica ainda é questionada. Para a otimização e viabilização comercial deste bioprocesso, estudos em fotobiorreatores (PernerNochta e Posten, 2007; Gao et al., 2017) vem sendo conduzidos com o auxílio de técnicas numéricas como a fluidodinâmica computacional (CFD) de forma a obter resultados de maior precisão.

O presente trabalho tem por objetivo avaliar a fluidodinâmica em um biorreator em escala de bancada, e verificar a sua adequação para o cultivo de microalgas, utilizando para tanto o código OpenFOAM, versão 4.1.

\section{MATERIAIS E MÉTODOS}

O processo em estudo consiste em um fotobiorreator do tipo erlenmeyer em escala de bancada, com 2 litros de volume, $26,8 \mathrm{~cm}$ de altura, $15,32 \mathrm{~cm}$ de diâmetro inferior e 4,13 cm de diâmetro superior (Figura 1a). Neste, o cultivo é realizado na presença da microalga Spirulina sp. LEB 18, onde um duto de alimentação de ar atua como dispersor de bolhas com uma vazão de $1 \mathrm{~L} / \mathrm{min}$. Para a simulação, o sistema foi tratado com um caso monofásico 
turbulento, em uma abordagem bidimensional axissimétrica. A fase simulada, que consiste em um resíduo utilizado como substrato proveniente de indústria cervejeira (trub filtrado), apresenta massa específica e viscosidade com valores de $1049 \mathrm{~kg} / \mathrm{m}^{3}$ e $0,0016784 \mathrm{~kg} / \mathrm{m} . \mathrm{s}$, respectivamente.

A modelagem adotada compreende a discretização e aplicação do método dos volumes finitos nas equações governantes da quantidade movimento e turbulência. Para modelar a turbulência, foi escolhido o modelo k-omega SST, uma vez que este fornece valores adequados para os campos turbulentos (Koerich e Rosa, 2016). A simulação dos resultados dos campos de velocidade, pressão e turbulência foram feitos primeiramente em estado estacionário, de modo a acelerar o avanço da solução: para a malha mais grosseira, observouse que o escoamento se desenvolvia até cerca de 130 interações. Posteriormente, a partir dos valores obtidos, o caso foi tratado como transiente, com cálculo de valores médios para a pressão e velocidade, até estes valores estabilizarem, o que ocorre após 10 segundos.

Como condições de contorno, foram definidas uma velocidade igual a $1,762 \mathrm{~m} / \mathrm{s}$ no duto de alimentação, e pressão atmosférica na saída superior do erlenmeyer. Para as paredes, foi adotada uma condição de não deslizamento para os campos de velocidade. Para as faces internas da fatia do erlenmeyer, foram inseridas condições de simetria, de modo a representar o erlenmeyer em sua totalidade (Figura 1b).

Figura 1 - (a) Fotobiorreator utilizado do experimento, (b) sua representação axissimétrica, e detalhes da malha elaborada (c) na entrada e (d) na saída.

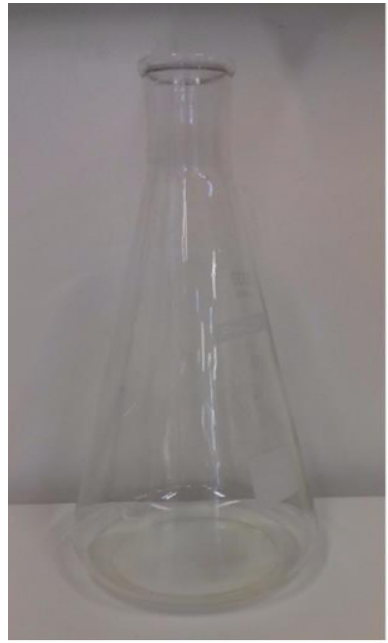

a

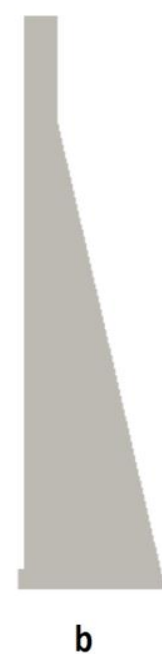

b

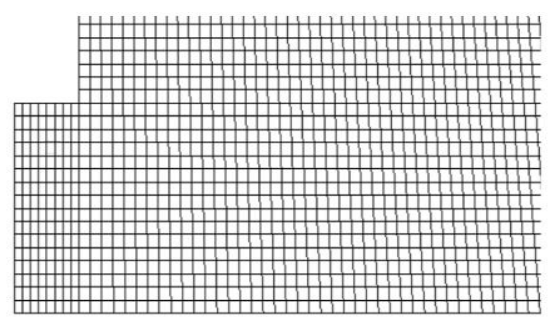

C

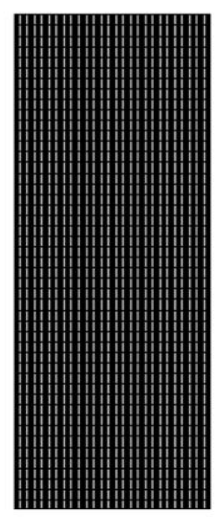

d

Para a estimativa da incerteza dos resultados numéricos obtidos, aplicou-se o procedimento GCI conforme descrito por Celik et al. (2008). Para tanto, foram efetuadas simulações em malhas com diferentes refinamentos, contendo 13 mil, 29 mil e 49,5 mil volumes de controle. Desta forma, obteve-se uma incerteza de $0,56 \%$ para a pressão. Para os campos de velocidade nas componentes " $x$ " e " $y$ ", calculada em 3 pontos distintos da malha, a incerteza foi significativamente maior, mas ainda assim a variação nas velocidades 
calculadas manteve-se menor do que $0,06 \mathrm{~m} / \mathrm{s}$. Desta forma, a malha contendo 49.568 volumes de controle foi considerada adequada para a condução do presente estudo. Detalhes dessa malha, nas regiões de alimentação e saída, podem ser vistos nas Figuras 1c e 1d, respectivamente.

\section{RESULTADOS E DISCUSSÃO}

Para a análise dos resultados preditos em simulação, foram feitas avaliações dos campos de velocidade, energia cinética, tensão cisalhante máxima e taxa de dissipação de energia cinética turbulenta. Valores médios e instantâneos foram considerados.

Como se pode observar na Figura 2, o campo de velocidade instantânea apresenta regiões com valores elevados, localizados próximo ao duto de alimentação, no vórtice formado na região inferior, e em uma pequena faixa na região intermediária da altura do frasco. Os resultados para a simulação em estado estacionário (Figura 2a), forneceram um esboço satisfatório, validando a estratégia de acelerar a solução antes de adotar cálculos transientes. Isto se confirma também pela visualização do comportamento do campo de velocidades entre os tempos de 5 segundos (Figura 2b) e 10 segundos (Figura 2c). O campo de velocidades médias (Figura 2d) destaca a constância de valores altos para regiões próximas ao duto de alimentação e oposto às simulações com valores instantâneos. Porém, os dados médios não ilustraram a formação de vórtice e a formação de uma região de alta velocidade na seção intermediária da altura do frasco erlenmeyer, o que indica que estes padrões de escoamento sejam transitórios.

Figura 2 - Campos de velocidade, para as simulações (a) estacionária, (b) transiente a 5 segundos, (c) transiente a 10 segundos, e (d) média transiente a 10 segundos.

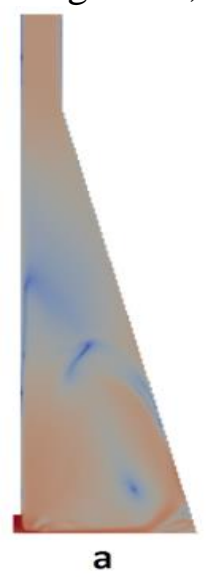

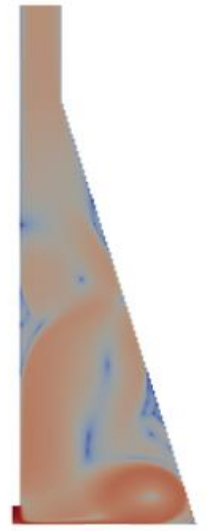

b

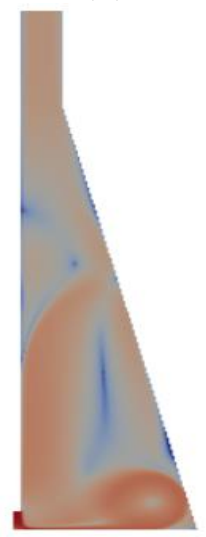

c

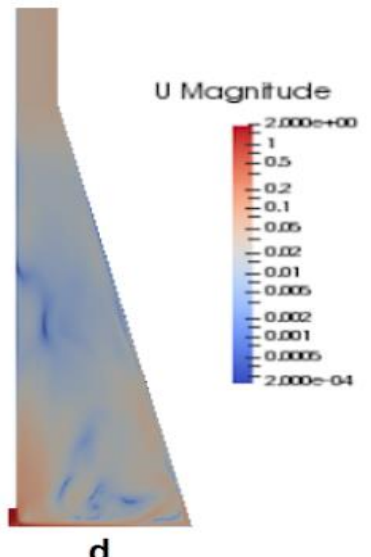

d

A energia cinética turbulenta favorece a mistura entre o substrato e microalga. Com base nos resultados obtidos em simulação (Figura 3), puderam-se identificar regiões de alta mistura, localizados no duto de alimentação e na parte externa do vórtice formado pelo campo de velocidade, conforme observado na Figura 2. Desde o final do campo obtido para estado estacionário (Figura 3a) até o último tempo transiente (Figura 3c), percebe-se que o campo turbulento se desenvolveu de modo a preencher todo o volume do erlenmeyer com um valor significativamente maior que a solução fornecida pelo caso estacionário. Identificam-se 
também regiões de mistura pobre em alguns locais do frasco, onde a energia cinética turbulenta possui valores mínimos devido à condição de não deslizamento (velocidade nula) das paredes.

Figura 3 - Campos de energia cinética turbulenta, para as simulações (a) estacionária, (b) transiente a 5 segundos, (c) transiente a 10 segundos.
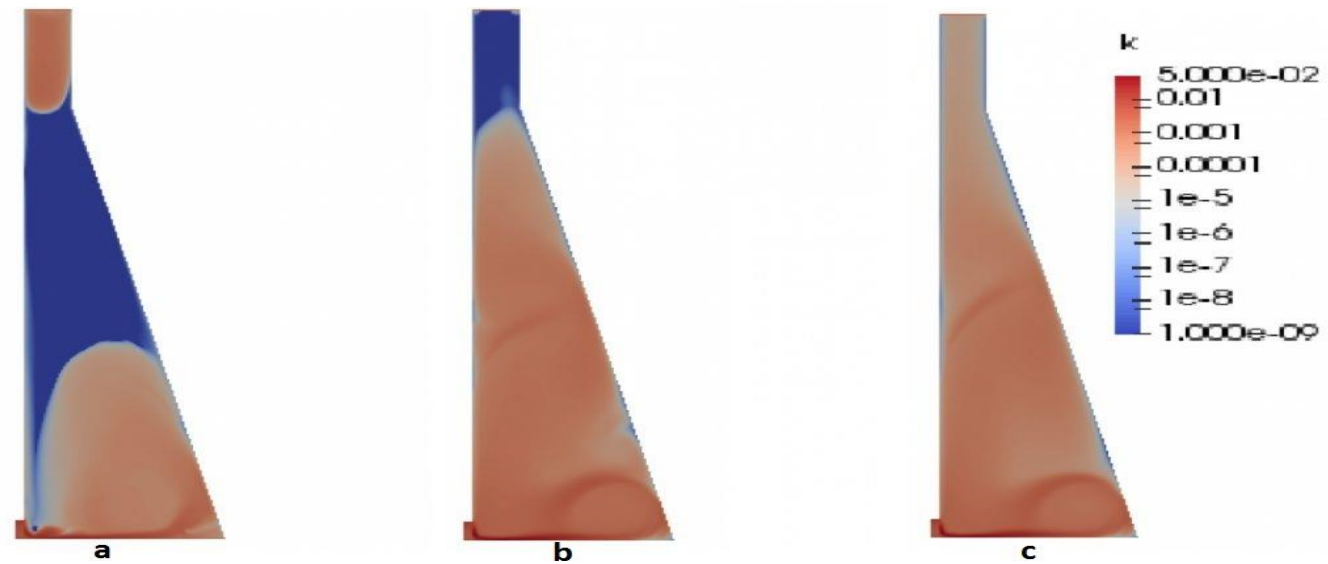

Os campos de tensão cisalhante manifestaram-se de maneira a reproduzir comportamento semelhante ao das variáveis já discutidas. A solução do caso estacionário (Figura 4a) forneceu valores iniciais consistentes e satisfatórios para o início da simulação transiente. Os valores instantâneos obtidos a 5 segundos (Figura 4b) e 10 segundos (Figura 4c) revelam a intensificação da tensão nas regiões externas do vórtice e próximas a parede. Os valores médios (Figura 4d) forneceram valores ainda menores para tensão cisalhante, exceto na parede e alimentação.

Figura 4 - Campos de tensão cisalhante, para as simulações (a) estacionária, (b) transiente a 5 segundos, (c) transiente a 10 segundos, (d) média transiente a 10 segundos.
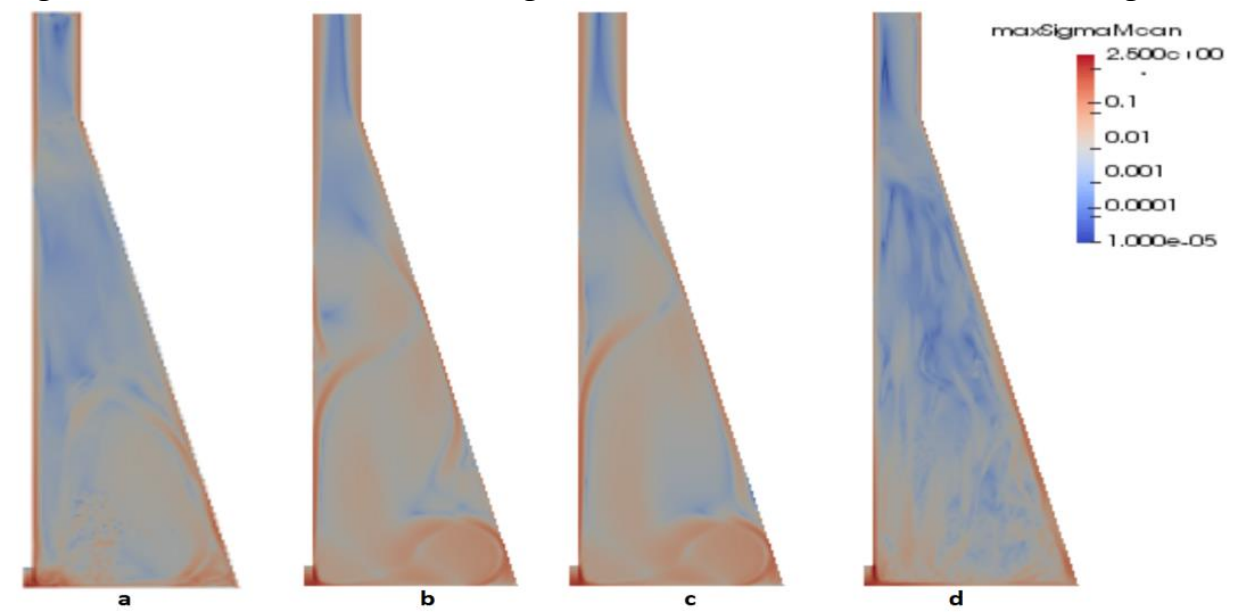
A análise dos campos de tensão indica regiões nas quais podem haver danos às células, reduzindo a atividade microbiana (Maurina et al., 2014). Segundo Michels et al. (2010), os valores entre 1 e 1,3 Pa representam o limite para que a tensão cisalhante prejudique o crescimento microalgal da Chaetoceros muelleri e de acordo com Mitsuhashi et al. (1994), o tricoma (apêndices epidérmicos que atuam na proteção da célula) da microalga Spirulina sp. ligeiramente decresce quando exposta a tensões acima de $0,2 \mathrm{~Pa}$ e, significativamente quando atinge valores superiores a 0,4 $\mathrm{Pa}$. Diante dos dados encontrados na literatura, pode-se afirmar que de acordo com os resultados obtidos em simulação, ilustrados nas Figuras $4 \mathrm{c}$ e $4 \mathrm{~d}$, a maior parte do frasco erlenmeyer está com tensões cisalhantes abaixo do limite indicado em literatura.

O campo da taxa de dissipação de energia cinética turbulenta descreveu valores coerentes ao campo de energia cinética turbulenta previamente discutido, indicando aumento das variáveis pela totalidade do volume.

Figura 5 - Campos de taxas de dissipação de energia cinética turbulenta, para as simulações (a) estacionária, (b) transiente a 5 segundos, (c) transiente a 10 segundos.
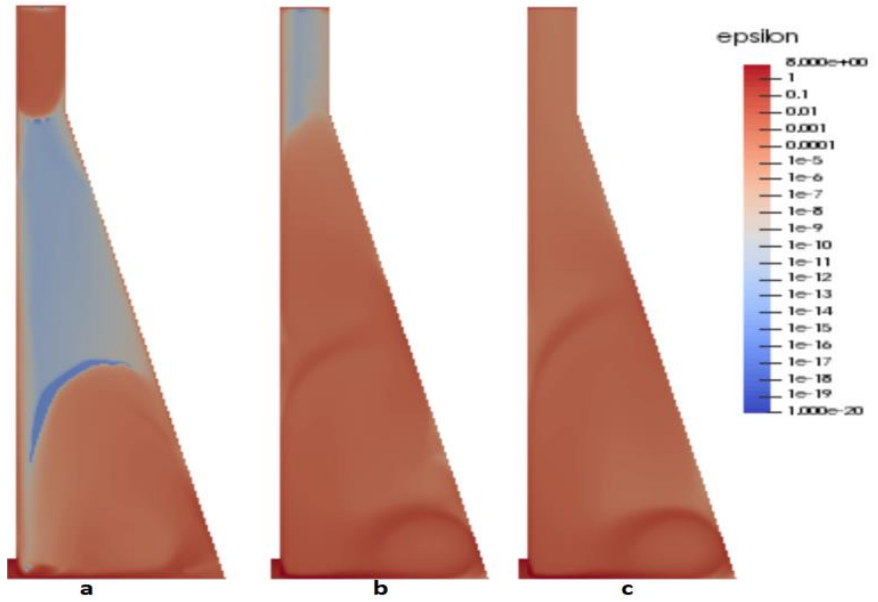

Diferentemente das células animais que apresentam fragilidade celular, as células da microalga dinoflagelada estudada por Gallardo-Rodriguez et al. (2016) resistiram a valores de taxa dissipada acima de $10^{10} \mathrm{~W} / \mathrm{m}^{3}$. Os resultados dos campos expostos, para ambos casos estacionário e transiente, apresentaram valores satisfatórios quando comparados com os dados propostos pela literatura, tendo como valor máximo $8.10^{-1} \mathrm{~W} / \mathrm{m}^{3}$.

\section{CONCLUSÃO}

Neste estudo foram avaliados os campos de velocidade, energia cinética turbulenta e tensão cisalhante, através da condução de simulações monofásicas em geometria axissimétrica, calculadas inicialmente em estado estacionário e então progredindo para o transiente. A análise de incerteza das malhas indicou que uma malha contendo 49568 volumes de controle é adequada. Fenômenos como a vorticidade, identificados nos campos de velocidade, influenciam diretamente a energia cinética turbulenta e por consequência a eficiência de contato entre substrato e microalga. Por outro lado, destaca-se também, que com o aumento da velocidade, interfere na tensão cisalhante exercida pelo fluido. Exceto pela região de 
alimentação, as demais regiões apresentaram condições hidrodinâmicas favoráveis para o crescimento microalgal.

\section{REFERENCIAS}

CELIK, I. B.; GHIA, U.; ROACHE, P. J.; FREITAS, C. J.; COLEMAN, H.; RAAD, P. E. Procedure for estimation and reporting of uncertainty due to discretization in CFD applications. J. of Fluids Eng., v. 130, n. 7, 2008.

GALLARDO-RODRIGUEZ，J. J.; LÓPEZ-ROSALES， L.; SÁNCHEZ-MIRÓN， A.; GARCIA-CAMACHO, F.; MOLINA-GRIMA, E.; CHALMERS, J. J. New insights into shear-sensitivity in dinoflagellatte microalgae. Biores. Technol., v. 200, p. 699-705, 2016.

GAO, X.; KONG, B.; VIGIL, D. Comprehensive computational model for combining fluidhydrodynamics, light transport and biomass growth in a Taylor vortex algal photobioreactor: Lagrangian approach. Biores. Technol., v. 224, p. 523-530, 2016.

KOERICH, D. M.; ROSA, L. M. Numerical evaluation of the low Reynolds turbulent flow behavior in a bioreactor. Int J. of Simul and Process Model., v. 11, n. 1, p. 66-75, 2016.

MAURINA, G. Z.; ROSA, L. M.; BEAL, L. L.; BALDASSO, C.; GIMENEZ, J. R.; TORRES, A. P.; SOUSA, M. P. Effect of internal recirculation velocity in an anaerobic sequencing batch reactor (ASBR). Brazil. J. of Chem Eng., v. 31, n. 4, p.895-903, 2014.

MICHELS, M. H. A.; VAN DER GROOT, A. J.; NORSKER, N.-H.; WIJFFELS, R. H. Effects of shear stress on the microalgae Chaetoceros muelleri. Bioproc. and Biosys. Eng., v. 33, n. 8, p. 921-927, 2010.

MILLEDGE, J. J. Commercial application of microalgae other than as biofuels: a brief review. Rev. in Environ. Sci. and Biotechnol., v. 10, p. 30-41, 2011.

MITSUHASHI, S.; FUJIMOTO, M.; MURAMATSU, H.; TANISHITA, K. Effect of simple shear flow on photosynthesis rate and morphology of micro algae. Acta Astronautica, v. 33, p. 179-187, 1994.

NIKOLAOU, A.; BOOTH, P.; GORDON, F.; YANG, J.; MATAR, O.; CHACHUAT, B. Multi-physics modeling of light-limited microalgae growth in raceway ponds. IFACPapersOnline v. 49, n. 26, p. 324-329, 2016.

PERNER-NOCHTA, I.; POSTEN, C. Simulations of light intensity variation in photobioreactors. J of Biotechnol., v. 131, n. 3, p. 276-285, 2007. 